\title{
Correction to: Sequence variation in ribosomal DNA and in the nuclear $h s p 90$ gene of Pratylenchus penetrans (Nematoda: Pratylenchidae) populations and phylogenetic analysis
}

\author{
Elena Fanelli • Alberto Troccoli • Francesco Capriglia • Giuseppe Lucarelli • Nicola Vovlas • \\ Nicola Greco • Francesca De Luca
}

Published online: 10 May 2018

(C) Koninklijke Nederlandse Planteziektenkundige Vereniging 2018

\author{
Correction to: Eur J Plant Path. \\ https://doi.org/10.1007/s10658-018-1480-1 \\ Results
}

Paragraph: Ribosomal sequence characterization.

Fig. 1 This figure is missing and the legend of Fig. 1 is erroneously labeled as Fig. 2, and associated to the phylogenetic tree which corresponds to Fig. 2

-Legend of Fig. 2: The correct legend for Fig. 2 reported in the paper is erroneously labeled as Fig. 3. The correct legend should be read:

Fig. 2 Phylogenetic tree of ITS containing region describing the evolutionary relationships among different

The online version of the original article can be found at https://doi.org/10.1007/s10658-018-1480-1

E. Fanelli $\cdot$ A. Troccoli $\cdot$ F. Capriglia $\cdot$ N. Vovlas $\cdot$

N. Greco · F. De Luca $(\bowtie)$

Istituto per la Protezione Sostenibile delle Piante (IPSP), Consiglio Nazionale delle Ricerche (CNR), S.S. di Bari, Via G. Amendola, 122/D, 70126 Bari, Italy

e-mail: francesca.deluca@ipsp.cnr.it

G. Lucarelli

Hortoservice - Servizi Tecnici in Orticoltura via San Pietro,

3-70016 Noicattaro, BA, Italy geographical populations of Pratylenchus penetrans and other Pratylenchus species, using Maximum Likelihood (ML) method. Branch lengths are proportional to the distances as derived from the distance matrix obtained using the GTR method with the invariant site plus gamma options. Numbers at nodes indicate bootstrap values

-Legend of Fig. 3: The correct legend for Fig. 3 reported in the paper is erroneously labeled as Fig. 4. The correct legend should be read:

Fig. 3 Phylogenetic tree of D2-D3 expansion domains describing the evolutionary relationships among different geographical populations of Pratylenchus penetrans and other Pratylenchus species, using Maximum Likelihood (ML) method. Branch lengths are proportional to the distances as derived from the distance matrix obtained using the GTR method with the invariant site plus gamma options. Numbers at nodes indicate bootstrap values

Legend of Fig. 4: This legend is missing in Fig. 4. The correct legend should be read:

Fig. 4 Phylogenetic tree of the hsp90 gene sequence dataset describing the evolutionary relationships within populations of Pratylenchus penetrans and other Pratylenchus species using Maximum Likelihood (ML) method. Branch lengths are proportional to the distances as derived from the distance matrix obtained using the GTR method with the invariant site plus gamma options. Numbers at nodes indicate bootstrap values 


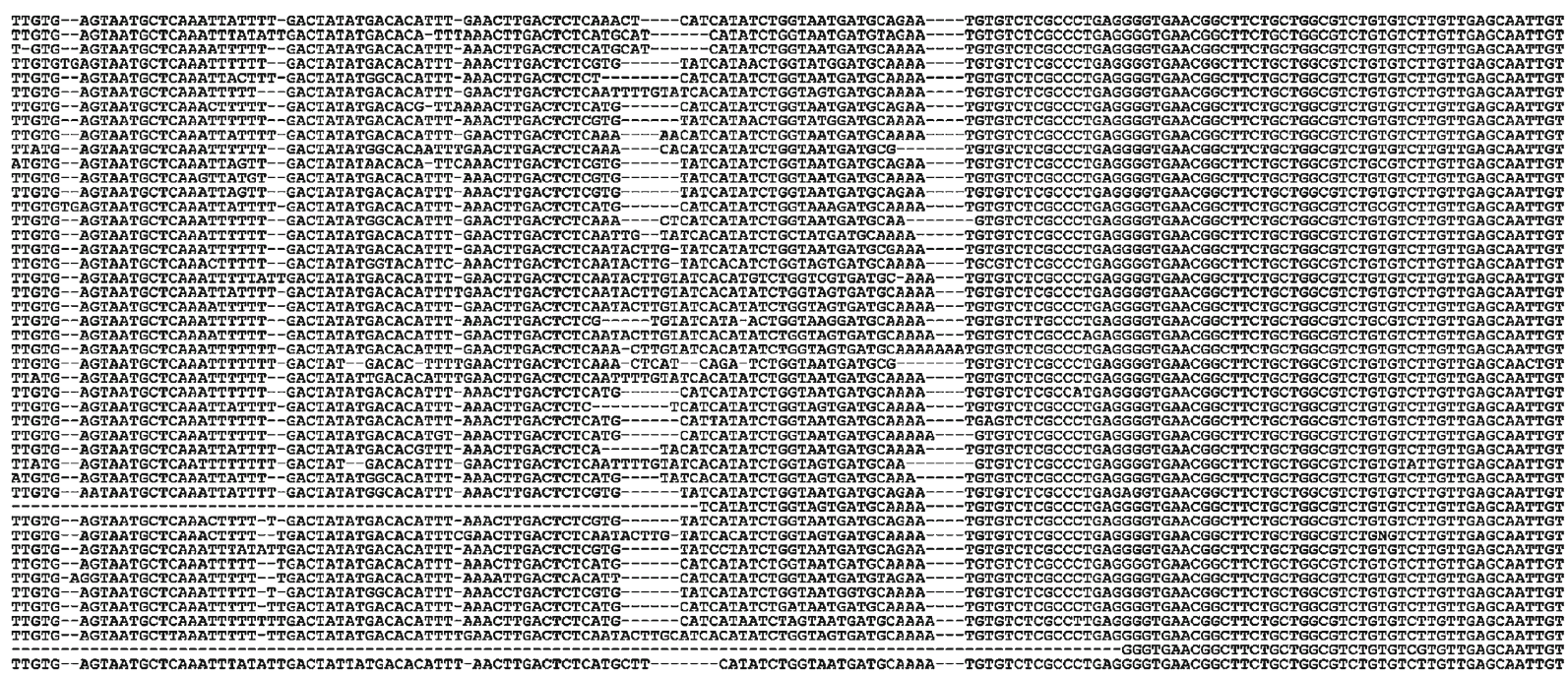

Fig. 1 